\title{
Fluorescence-based remote irradiation sensor in liquid- filled hollow-core photonic crystal fiber
}

\author{
R. Zeltner ${ }^{1,2}$, D.S. Bykov ${ }^{1}$, S. Xie ${ }^{1}$, T.G. Euser ${ }^{3,1}$ and P. St.J. Russell ${ }^{1,2}$ \\ ${ }^{1}$ Max Planck Institute for the Science of Light and ${ }^{2}$ Department of Physics, University of Erlangen- \\ Nuremberg, Guenther-Scharowsky-Str. 1, 91058 Erlangen, Germany \\ ${ }^{3}$ Cavendish Laboratory, University of Cambridge, J. J. Thomson Avenue, Cambridge CB3 0HE, UK
}

We report an irradiation sensor based on a fluorescent 'flying particle' that is optically trapped and propelled inside the core of a water-filled hollow-core photonic crystal fiber. When the moving particle passes through an irradiated region, its emitted fluorescence is captured by guided modes of the fiber core and so can be monitored using a filtered photodiode placed at the fiber end. The particle speed and position can be precisely monitored using in-fiber Doppler velocimetry, allowing the irradiation profile to be measured to a spatial resolution of $\sim 10 \mu \mathrm{m}$. The spectral response can be readily adjusted by appropriate choice of particle material. Using dye-doped polystyrene particles, we demonstrate detection of green (532 nm) and ultraviolet (340 nm) light. 
Fiber-based irradiation sensors are of great interest in applications such as nuclear science, chemical engineering and medicine. Typically, the tip of an optical fiber is coated with a suitable luminescent material, and the emitted radiation is collected by the fiber core and transmitted to the diagnostics. While this method enables detection from the ultraviolet (UV) to the x-ray spectral regions [1-4], spatially resolved measurements are cumbersome and difficult, especially in hazardous environments. In addition, the detectable spectral range is limited by the properties of the luminescent material, and material degradation (e.g., photo-darkening) can severely limit applications when the irradiation levels are high. Hollow-core photonic crystal fiber (HC-PCF), on the other hand, is much more robust against radiation damage. A short length of HC-PCF was recently used to shield an optically trapped microparticle during a fluorescence experiment, without exploiting the optical guidance properties of the HC-PCF [5].

Here we report a reconfigurable fiber sensor that combines damage resistance with a high spatial resolution. It is based on a fluorescent or radioluminescent 'flying-particle' that is optically trapped and propelled into a hollow-core photonic crystal fiber (HC-PCF) [6]. Because the particle is guided close to the center of the core, its fluorescence is readily captured by guided modes and so can be monitored from the fiber end (this was previously shown using lateral laser tweezers to trap a single dye-doped microparticle at a fixed position inside a HC-PCF [7]). By adjusting the balance of backwards and forwards laser power, the particle can be moved to and fro, permitting an irradiation profile to be resolved with high positional accuracy over distances which are only limited by the waveguide losses. 
An additional advantage of this system is that the particle is protected by the glass cladding of the fiber, shielding the particle from unwanted external influences such as gas flow or chemical attack, and making it suitable for sensing in harsh environments. The hollow core also enables the use of intense UV-light [8] or highly radioactive sources, where conventional solid-glass cores rapidly darken due to radiation damage $[9,10]$. The set-up is highly reconfigurable, allowing for example the spectral response to be adjusted simply by launching particles with different absorption and emission spectra.

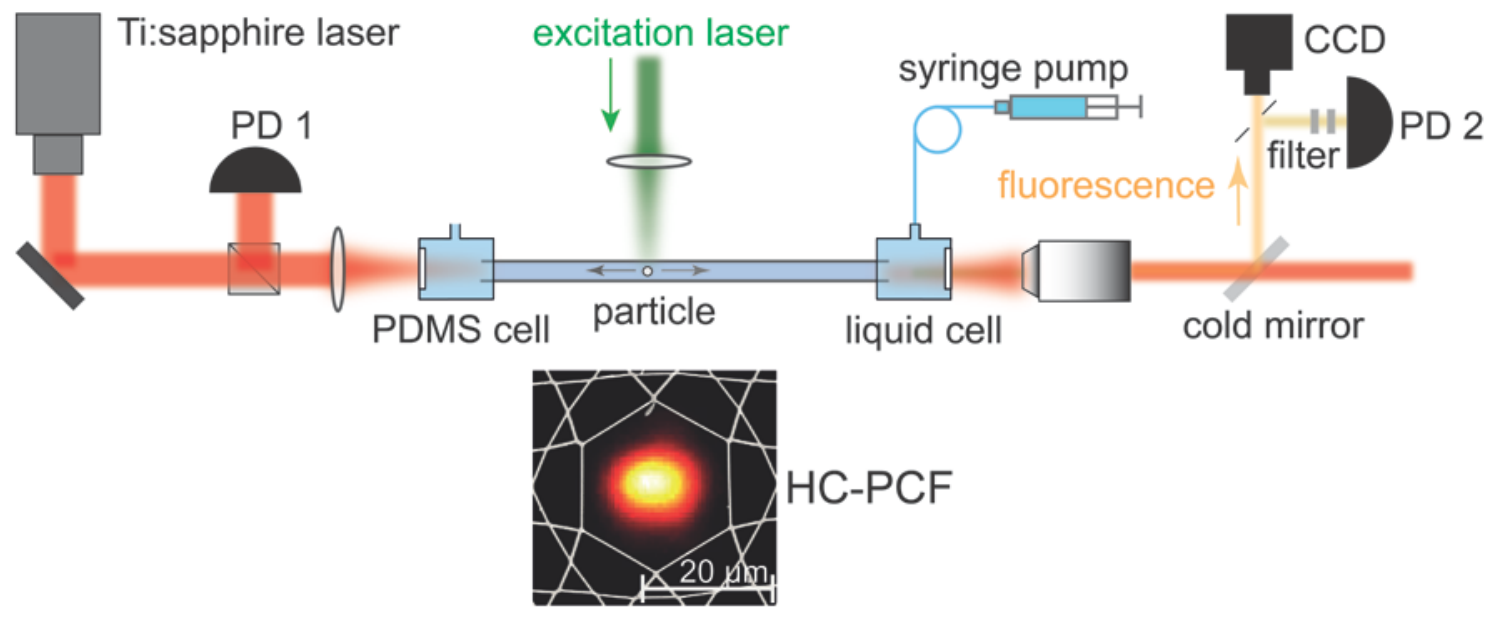

Fig. 1: Schematic of set-up. A fluorescent microparticle is optically propelled along the HC-PCF. Its fluorescence is guided to the fiber end, spectrally filtered, and detected by a sensitive Si photodiode (PD 2, Thorlabs PDF10A). A $532 \mathrm{~nm}$ laser diode and a $340 \mathrm{~nm}$ light emitting diode (LED) were used as transverse irradiation sources. (bottom part) A scanning electron micrograph of the kagomé-PCF superimposed with an optical micrograph of the measured near-field profile at $785 \mathrm{~nm}$ (a similar field pattern is seen for excitation and emission wavelengths).

A schematic of the experimental set-up is shown in Fig. 1. One end of a 25-cm-long waterfilled kagomé-style hollow-core PCF (kagomé-PCF) with a core diameter of $21.5 \mu \mathrm{m}$ is clamped inside a liquid cell connected to a syringe pump, while the other end is placed 
inside a polydimethylsiloxane (PDMS) chamber with a volume of $\sim 50 \mu \mathrm{L}$. Light is launched into the fiber through glass windows in the liquid cell and the PDMS chamber. A procedure similar to that described in [11] is used to launch a fluorescent polystyrene microparticle of diameter $\sim 3.2 \mu \mathrm{m}$ (Thermo Fisher Scientific Inc., Fluoro-Max red) from the PDMS chamber into the fiber core. Once inside fiber, the particle was propelled by a Ti:sapphire laser operating at $785 \mathrm{~nm}$, while its speed and position were monitored by Doppler velocimetry [12], mixing light back-scattered from the particle and the fiber endface at PD1. Since the modal refractive indices are within $0.1 \%$ of the index of water, the Doppler shift is negligibly affected by scattering into higher order modes. The fiber loss at this wavelength is $\sim 20 \mathrm{~dB} / \mathrm{m}$ and is mainly due to water absorption. The typical guidance velocity was $200 \mu \mathrm{m} / \mathrm{s}$ for an optical power of $100 \mathrm{~mW}$, yielding an optical mobility of $2 \mathrm{~mm} \cdot \mathrm{s}^{-1} \mathrm{~W}^{-1}$. The particle can be held stationary or moved backwards by applying a liquid counterflow via the syringe pump. In one location the fiber was transversely illuminated by a weakly focused laser diode emitting $5.5 \mathrm{~mW}$ at $532 \mathrm{~nm}$, close to the excitation peak of the fluorescent dye $\left(\lambda_{\mathrm{ex}}=542 \mathrm{~nm}\right)$. As the particle passes through this region, part of its fluorescence (emission peak at $\lambda_{\mathrm{em}}=612 \mathrm{~nm}$ ) is captured by the kagomé-PCF modes and guided to the fiber end, where it is filtered out by a combination of spectral short and long pass filters and detected by a photodiode (PD 2). The fiber loss at this emission wavelength is $\sim 15 \mathrm{~dB} / \mathrm{m}$. 

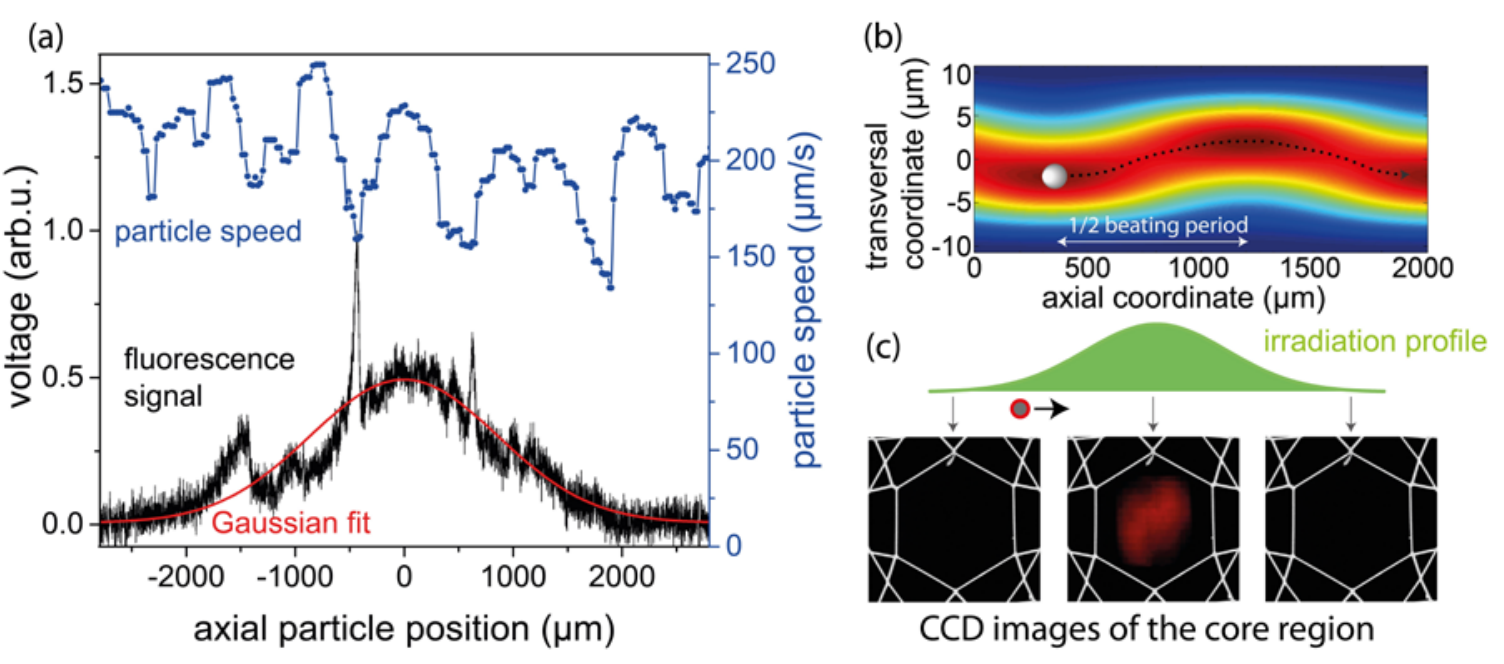

Fig. 2: (a) Measured Gaussian-like intensity profile projected on to the fiber (black curve) and particle speed (dotted blue curve). The peaks at axial positions $-1500 \mu \mathrm{m},-500 \mu \mathrm{m}$ and $500 \mu \mathrm{m}$ are induced by transverse displacement of the particle from the core center due to intermodal beating (see below). The measured shape is accurately approximated by a Gaussian fit with $1 / \mathrm{e}^{2}$ half-width $1721 \mu \mathrm{m}$. (b) Calculated intensity distribution of the fiber, caused by intermodal beating. (c) Typical CCD-pictures of the fiber core region when a particle was moved through the focus of the excitation laser.

The $532 \mathrm{~nm}$ excitation beam was weakly focused using a cylindrical lens, creating a Gaussian-shaped intensity profile along the axis of the fiber. The resulting elongated intensity distribution ensured stable alignment of the excitation spot on the fiber, making it easier to compare the experimental results with the reference data taken with a beam profiler. Fig. 2(a) shows the fluorescence signal detected as a function of particle position relative to the center of the illumination spot. It can be seen that the observed signal accurately fits to a Gaussian profile with $1 / \mathrm{e}^{2}$ half-width of $1721 \pm 70 \mu \mathrm{m}$, in excellent agreement with the value obtained using the beam profiler (1714 $\mu \mathrm{m})$.

Although during alignment we tried to excite only the $\mathrm{LP}_{01}$ mode, the particle velocity showed periodic fluctuations caused by intermodal beating with the $\mathrm{LP}_{11}$ mode, which was 
weakly, but unavoidably, excited. As an example, Fig. 2(b) shows the calculated intensity distribution for a mixture of $10 \% \mathrm{LP}_{11}$ mode and $90 \% \mathrm{LP}_{01}$ mode, using expressions for the modal refractive indices from Marcatili and Schmelzer [13]. The in-coupling conditions were optimized such that the contribution from modes with order above $\mathrm{LP}_{11}$ is negligible. The beating causes periodic fluctuations in the transverse intensity distribution with axial position, causing the particles to follow a zig-zag trajectory along the fiber core. Interestingly, sharp peaks in fluorescence signal are detected at $-1500 \mu \mathrm{m},-500 \mu \mathrm{m}$ and $500 \mu \mathrm{m}$ in Fig. 2(a). These coincide with minima in the Doppler velocimetry data. We believe that this is related to the more central particle position in these regions, resulting in better coupling of the fluorescence to guided modes. The distance between the peaks $(\sim 1000 \mu \mathrm{m})$ corresponds well to one half the experimentally measured beat-length between the $\mathrm{LP}_{01}$ and $\mathrm{LP}_{11}$ modes $(\sim 1940 \mu \mathrm{m})$.

To further confirm that the signal is caused by fluorescence and not by scattered excitation light, the output mode profile was monitored with a CCD-camera while the particle moved through the irradiated region. The fluorescence signal was maximized by focusing the excitation laser onto the fiber using a convex spherical lens. To avoid detection of scattered excitation light, a $550 \mathrm{~nm}$ long pass filter was placed in front of the camera. Fig. 2(c) shows CCD frames obtained as the particle passed the illuminated spot. The spectrum of the red light in the core agrees with the expected fluorescence of the particle, indicating that the signal is due to fluorescence. As the emitted fluorescence can couple to several waveguide modes, the intensity profile appears as a superposition of different modes.

To determine the linearity of the sensor, the fluorescence signal was measured for a range of different excitation powers. To prevent saturation of the detector at high irradiation 
levels a neutral density filter ( $5 \mathrm{~dB}$ attenuation) was used to attenuate the signal; during evaluation of the data we corrected for this attenuation. Fig. 3(a) shows the detected fluorescence signal as a function of particle position relative to the center of the Gaussian focus of the convex spherical lens. The fluorescence peak is accurately described by a Gaussian function with $1 / \mathrm{e}^{2}$ half-width of $37.5 \mu \mathrm{m}$ (adjusted $\mathrm{R}^{2}$ - value larger than 0.93 [14]). The detector response (inset of Fig. 3(a)) is linear with a slope of $0.037 \mathrm{~V} . \mathrm{W}^{-}$ ${ }^{1} \mathrm{~cm}^{-2}$. The fluorescence signal reaching the detector was estimated to be $10 \mathrm{pW}$ for an excitation irradiance of $100 \mathrm{~W} / \mathrm{cm}^{2}$.
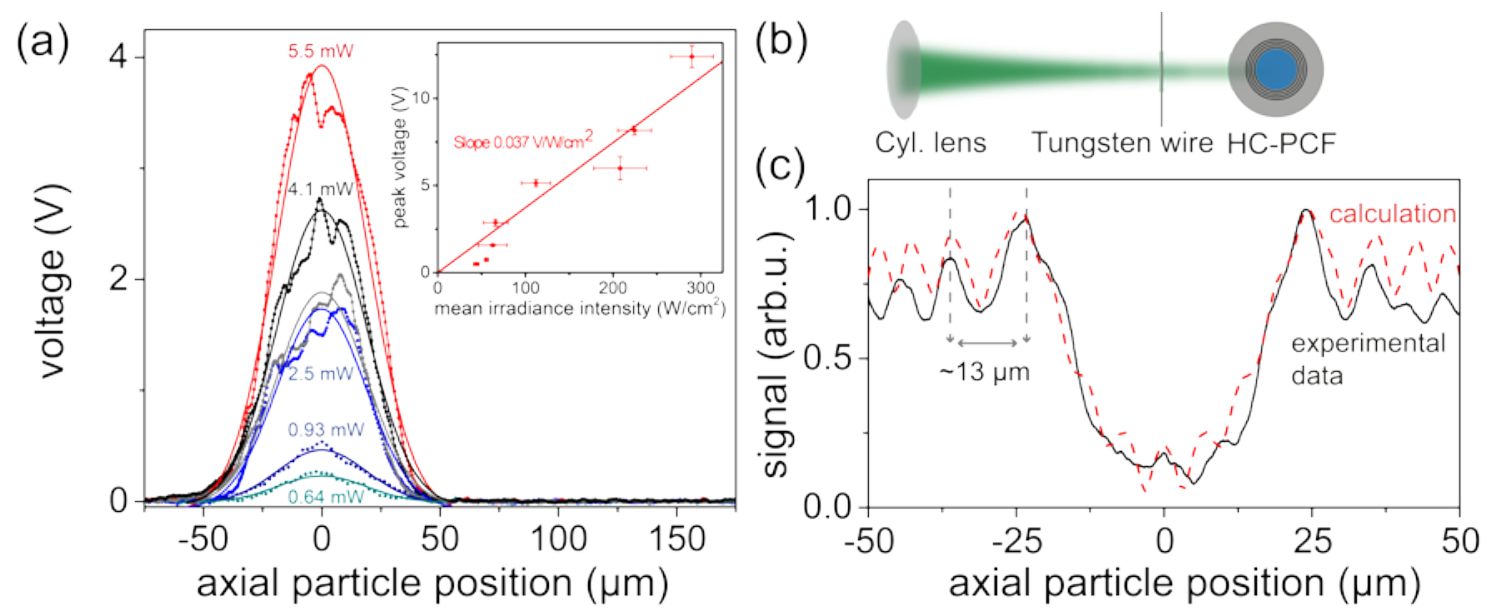

Fig. 3: (a) Detected fluorescence signal as a function of particle position for different optical powers. Inset: Dependence of the peak height on irradiation intensity. The error bars indicate the standard deviations of the measured peak height and the calculated irradiance intensity. (b) A diffraction pattern created by a tungsten wire was used to test the spatial resolution of the sensor. (c) The experimental data (black, solid curve) is in good agreement with the calculated intensity profile (red, dashed curve).

To test the spatial resolution of the sensor we created a diffraction pattern by placing a tungsten wire with a diameter of $26 \mu \mathrm{m}$ at a distance of $\sim 300 \mu \mathrm{m}$ away from the fiber core center. Fig. 3(c) shows the normalized measurement along with the expected pattern 
calculated from analytical diffraction theory for a cylindrical wire [15]. It can be seen that the first and the second order diffraction peaks are in good agreement with theory. For the higher order diffraction peaks, however, the increasing discrepancy may be explained by refraction of the excitation light in the cladding structure and inhomogeneities in the fiber coating (not taken into account in the calculations). The experimentally demonstrated spatial resolution was $\sim 13 \mu \mathrm{m}$, given by the distance between first and second order peaks. We note that the spatial resolution is ultimately limited only by the diameter of the particles.

Finally, the spectral flexibility of the sensor is demonstrated by replacing the particle with a $\sim 5 \mu \mathrm{m}$ diameter polystyrene particle doped with an ultraviolet-sensitive dye ( $\lambda_{\text {ex }}=340$ $\mathrm{nm}, \lambda_{\text {em }}=525 \mathrm{~nm}$, Thermo Fisher Scientific Inc.). Due to the larger particle size the radiation pressure acting on the propelled microparticle is larger than in the previous measurements, yielding a particle speed of $300 \mu \mathrm{m} / \mathrm{s}$ for the same guided optical power (optical mobility of $3 \mathrm{~mm} \cdot \mathrm{s}^{-1} \mathrm{~W}^{-1}$ ). The fiber was illuminated with a focused LED emitting at $\sim 340 \mathrm{~nm}$. Since the polymer coating absorbs in the UV-region, it was removed over the illuminated portion of the fiber. Analogously to the previous measurement, the particle was optically propelled through the irradiated region. Fig. 4 shows the detected signal for two different excitation powers. The focal spot size of the LED $\left(\sim 0.9 \mathrm{~mm}^{2}\right)$ is much larger than that of the $532 \mathrm{~nm}$ laser diode, resulting in a fluorescence signal that is only a few times above the detector noise level. Data taken at two different powers suggests a linear response (inset). Defining the noise level as three times the standard deviation of the detector output voltage in the absence of any signal ( $3 \sigma$ level) we estimate the detection limit to be $\sim 1$ $\mathrm{Wcm}^{-2}$ in the experimental configuration. 


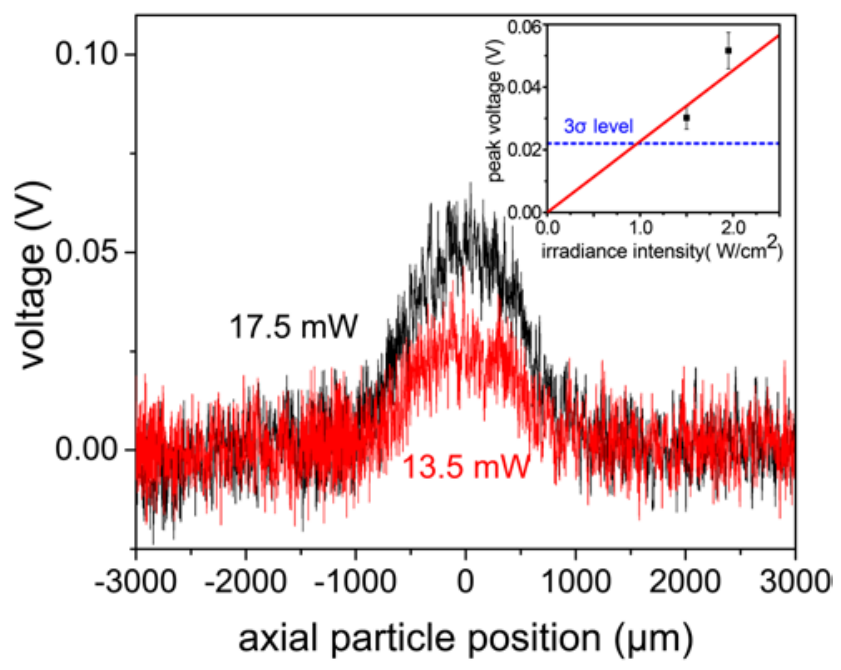

Fig. 3: Detected fluorescence signal from the UV sensitive microparticle as a function of particle position for two different LED powers at $340 \mathrm{~nm}$. Inset: the slope $\left(0.023 \mathrm{~V} . \mathrm{W}^{-1} \mathrm{~cm}^{-2}\right)$ and the noise level $(\sim 0.022 \mathrm{~V})$ indicate that irradiation intensities as small as $\sim 1 \mathrm{Wcm}^{-2}$ can be detected in the near UV.

In conclusion, fluorescent microparticles optically guided inside the core of a HC-PCF can be used to probe irradiation remotely with spatial resolution determined by the particle size. The spectral range of operation can be conveniently changed by employing microparticles with different spectral absorption and emission. This offers unique flexibility, potentially allowing one to probe a spectral range of several hundred nanometers with one sensor. As both the trapping light and the signal are guided in the liquid-filled core, the sensor is unaffected by photo-darkening, making it suitable for the use in spectral regions where solid-core step-index fibers are unsuitable. Another interesting feature of the system is that both the resolution and the sensitivity of the sensor can be dynamically adapted by changing the size of the particles: smaller particles provide higher spatial resolution, while larger particles absorb more of the irradiance and therefore provide higher sensitivity. 
The maximum fiber length in the current configuration is limited by water absorption to 1 m. This can be easily extended to several meters by shifting the guidance laser to a shorter wavelength or by using an evacuated kagomé-PCF. Finally, the influence of intermodal beating can be overcome using a pulsed laser for trapping [6] or by employing an effectively single-mode "single-ring" HC-PCF [16,17]. The use of such a fiber, with its much simpler cladding structure, would also reduce scattering and reflection in the cladding, enhancing the performance of the sensor.

\section{ACKNOWLEDGMENTS}

Richard Zeltner acknowledges funding from the Cluster of Excellence 'Engineering of Advanced Materials’ (www.eam.uni-erlangen.de) at the University of ErlangenNuremberg.

\section{REFERENCES}

1. C. Fitzpatrick, C. O’Donoghue, J. Schöbel, B. Bastiaens, P. van der Slot, E. Lewis, and D. Pendrill, "A large core polymer optical fibre sensor for x-ray dosimetry based on luminescence occurring in the cladding," Meas. Sci. Technol. 15, 1586-1590 (2004).

2. C. Fitzpatrick, C. O?Donoghue, and E. Lewis, "A novel multi-point ultraviolet optical fibre sensor based on cladding luminescence," Meas. Sci. Technol. 14, 1477 (2003). 
3. D. McCarthy, S. O’Keeffe, E. Lewis, D. G. Sporea, A. Sporea, I. Tiseanu, P. Woulfe, and J. Cronin, "Radiation Dosimeter Using an Extrinsic Fiber Optic Sensor," IEEE Sens. J. 14, 673-685 (2014).

4. M. McSherry, C. Fitzpatrick, and E. Lewis, "An optical fiber sensor for the detection of germicidal UV irradiation using narrowband luminescent coatings," IEEE Sens. J. 4, 619-626 (2004).

5. V. K. Shinoj and V. M. Murukeshan, "Hollow-core photonic crystal fiber based multifunctional optical system for trapping, position sensing, and detection of fluorescent particles," Opt. Lett. 37, 1607 (2012).

6. D. S. Bykov, O. A. Schmidt, T. G. Euser, and P. S. J. Russell, "Flying particle sensors in hollow-core photonic crystal fibre," Nat. Photonics 9, 461-465 (2015).

7. C. Rajapakse, F. Wang, T. C. Y. Tang, P. J. Reece, S. G. Leon-Saval, and A. Argyros, "Spectroscopy of 3D-trapped particles inside a hollow-core microstructured optical fiber," Opt. Express 20, 11232 (2012).

8. F. Gebert, M. H. Frosz, T. Weiss, Y. Wan, A. Ermolov, N. Y. Joly, P. O. Schmidt, and P. S. J. Russell, "Damage-free single-mode transmission of deep-UV light in hollowcore PCF," Opt. Express 22, 15388 (2014).

9. N. Yamamoto, L. Tao, and A. P. Yalin, "Single-mode delivery of $250 \mathrm{~nm}$ light using a large mode area photonic crystal fiber," Opt. Express 17, 16933 (2009).

10. W. P. Leung, M. Kulkarni, D. Krajnovich, and A. C. Tam, "Effect of intense and prolonged $248 \mathrm{~nm}$ pulsed-laser irradiation on the properties of ultraviolet-grade fused silica," Appl. Phys. Lett. 58, 551-553 (1991). 
11. T. G. Euser, M. K. Garbos, J. S. Y. Chen, and P. S. J. Russell, "Precise balancing of viscous and radiation forces on a particle in liquid-filled photonic bandgap fiber," Opt. Lett. 34, 3674 (2009).

12. M. K. Garbos, T. G. Euser, O. A. Schmidt, S. Unterkofler, and P. S. J. Russell, "Doppler velocimetry on microparticles trapped and propelled by laser light in liquidfilled photonic crystal fiber," Opt. Lett. 36, 2020-2022 (2011).

13. E. A. J. Marcatili and R. A. Schmeltzer, "Hollow Metallic and Dielectric Waveguides for Long Distance Optical Transmission and Lasers," Bell Syst. Tech. J. 43, 17831809 (1964).

14. N. H. Bingham and J. M. Fry, "Multiple Regression," in Regression, Springer Undergraduate Mathematics Series (Springer London, 2010), pp. 61-97.

15. R. F. Harrington, Time-Harmonic Electromagnetic Fields. (New York, 1961).

16. M. C. Günendi, P. Uebel, M. H. Frosz, and P. S. J. Russell, "Broad-band robustly single-mode hollow-core PCF by resonant filtering of higher order modes," ArXiv150806747 Phys. (2015).

17. P. Uebel, M. C. Günendi, M. H. Frosz, G. Ahmed, N. N. Edavalath, J.-M. Ménard, and P. S. J. Russell, "Broadband robustly single-mode hollow-core PCF by resonant filtering of higher-order modes," Opt. Lett. 41, 1961 (2016). 